\title{
Atmospheric Models for Aerocapture
}

\author{
C. G. Justus" and Aleta L. Duvall ${ }^{\dagger}$ \\ Morgan Research Corporation, Huntsville, AL, 35805 \\ and \\ Vernon W. Keller ${ }^{\ddagger}$ \\ NASA MSFC ED44, Marshall Space Flight Center, AL, 35812
}

\begin{abstract}
There are eight destinations in the Solar System with sufficient atmosphere for aerocapture to be a viable aeroassist option - Venus, Earth, Mars, Jupiter, Saturn and its moon Titan, Uranus, and Neptume. Engineering-level atmospheric models for four of these targets (Earth, Mars, Titan, and Neptune) have been developed for NASA to support systems analysis studies of potential future aerocapture missions. Development of a similar atmospheric model for Venus has recently commenced. An important capability of all of these models is their ability to simulate quasi-random density perturbations for Monte Carlo analyses in developing guidance, navigation and control algorithms, and for thermal systems design. Similarities and differences among these atmospheric models are presented, with emphasis on the recently developed Neptune model and on planned characteristics of the Venus model. Example applications for aerocapture are also presented and illustrated. Recent updates to the Titan atmospheric model are discussed, in anticipation of applications for trajectory and atmospheric reconstruct of Huygens Probe entry at Titan.
\end{abstract}

\section{Nomenclature}

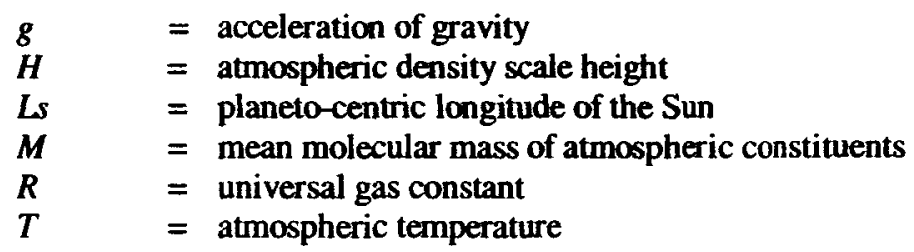

\section{Introduction}

E NGINEERING-LEVEL atmospheric models have been developed (or are under development) for five of the jeight possible Solar system destinations where aerocapture could be used. These include Global Reference Atmospheric Models (GRAMs) for Earth (GRAM-99) ${ }^{1,2}$, Mars (Mars-GRAM 2001) ${ }^{3-6}$, Titan (Titan-GRAM) ${ }^{7}$, Neptune (Neptune-GRAM), and Venus-GRAM (under development). Significant differences in physical characteristics of these planetary atmospheres, and significant differences in amount of available data on which to base engineering-level models of these atmospheres, means that detailed characteristics differ significantly among these models.

Earth-GRAM is based on climatology assembled from extensive observations by balloon, aircraft, ground-based remote sensing, sounding rockets, and satellite remote sensing. Details are provided in the GRAM user's guide'. Mars-GRAM is based on climatologies of General Circulation Model (GCM) output, with details given in the MarsGRAM user's guide. ${ }^{3}$. Mars-GRAM has been validated ${ }^{46}$ by comparisons against observations made by Mars Global Surveyor, and against output from another Mars GCM. In contrast, data from which to build Titan-GRAM

\footnotetext{
- Senior Principal Scientist, Environments Group, MSFC ED44/Morgan Research, Senior Member (formerly with Computer Sciences Corporation).

$\dagger$ Senior Software Engineer, Environments Group, MSFC ED44/Morgan Research, Member (formerly with Computer Sciences Corporation).

₹ Aerospace Engineer, Environments Group, MSFC ED44, Senior Member.
} 
and Neptune-GRAM are more limited, coming primarily from Voyager observations and limited ground-based stellar occultation measurements. Titan-GRAM is based on data summarized in Ref. 9, while Neptune-GRAM was built from summaries of data contained in Ref. 10. For Venus, a substantial amount of data has been collected from orbiters and entry probes. These have been summarized in the Venus International Reference Atmosphere (VIRA) ${ }^{11}$, which forms the basis for Venus-GRAM (under development).

Figure 1 shows the wide variety of temperature profiles encountered among the planets and Titan. For Earth, Venus, Mars, and Titan, height is measured from a reference surface (mean sea level on Earth). On Neptune, height is measured above the level at which pressure is one bar (Earth normal sealevel pressure). All of the planets exhibit a troposphere region, where temperature decreases with altitude, indicative of heat flow upward from the surface (on average). All of the planets exhibit a thermosphere region, where (on average) temperature increases with altitude, because of absorption of heat flux from the Sun as it penetrates into the atmosphere. All of the planets have stratospheres, where temperature decrease above the surface diminishes, and remains relatively constant until the base of the thermosphere (Earth being the exception to this, where the presence of ozone and resultant atmospheric heating produces a local temperature maximum in Earth's stratospheremesosphere region).

For interest in aerocapture or aerobraking, atmospheric density is the most important parameter. Fig. 2 compares density profiles on the planets and Titan. Vertical dashed lines in Fig. 2 indicate typical density values at which aerocapture or aerobraking operations would occur. Intersections of the aerocapture dashed line with various density curves shows that aerocapture would occur at a wide range of altitudes at the various destinations, varying from about $50 \mathrm{~km}$ at Mars to about $300 \mathrm{~km}$ at Titan. Aerobraking at Earth, Mars, and Venus would take place near, and just above, the 100 $\mathrm{km}$ level, while at Neptune and Titan, aerobraking would be done near 550 to 750 $\mathrm{km}$, respectively.

Figure 2 shows that density decreases fairly

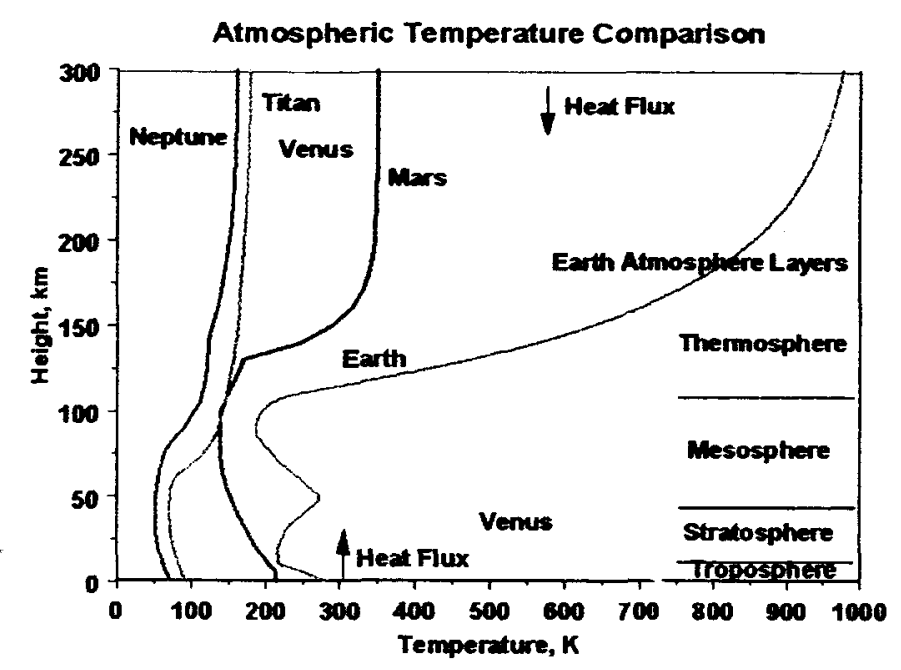

Figure 1: Comparison of temperature profiles among the planets and Titan.

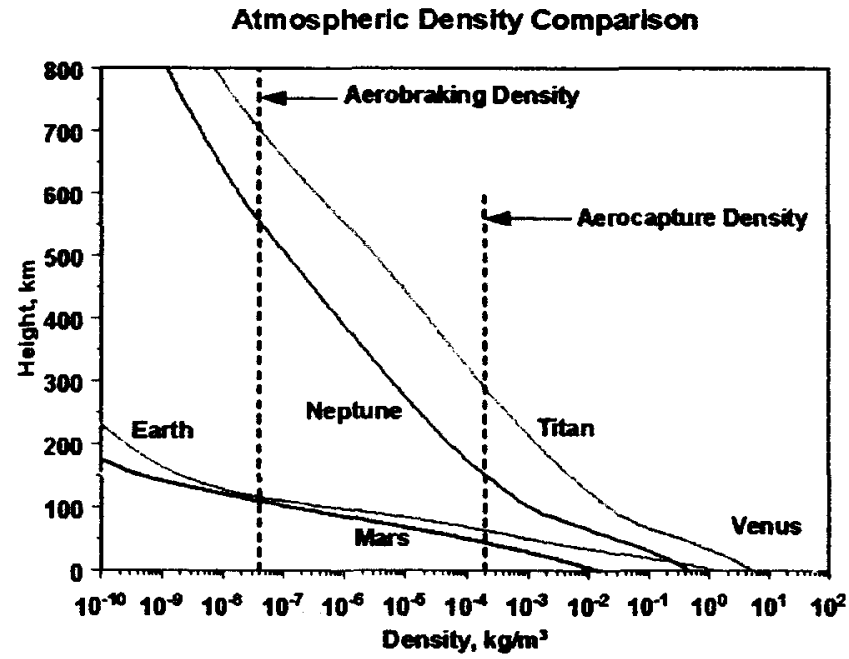

Figure 2: Comparison of density profiles among the planets and Titan

rapidly with altitude for the terrestrial planets (Venus, Earth, Mars), while it decreases rather slowly for Neptune and Titan. This effect is explained by differences in density scale height, $H$, for the various planets and Titan. Density decreases rapidly with altitude if $H$ is small, while it decreases slowly if $H$ is large. $H$ is proportional to pressure scale height [ $R T /(M g)]$. For the terrestrial planets, molecular mass is large $(M=29-44)$, so $H$ is small. On Neptune, $H$ is large because $M$ is small in its hydrogen-helium atmosphere $(M=2)$. For Titan, $H$ is large, despite its high molecular mass $(M \approx 29)$, because of its low gravity. 


\section{Basis for the Atmospheric Models}

In Earth-GRAM, Mars-GRAM, and Venus-GRAM, input values for date, time, latitude, longitude etc. are used to calculate planetary position and solar position, so that effects of latitude variation, and seasonal and time-of-day variations can be computed explicitly. A simplified approach is adopted in Titan-GRAM and Neptune-GRAM whereby these effects (as well as effects of relatively large measurement uncertainties for these planets) are represented within a prescribed envelope of minimum-average-maximum density versus altitude. Figure 3 shows this envelope for Titan, for which engineering atmospheric model data developed for Huygens entry probe' are used. For Neptune, data from Ref. 10 are employed, to generate a comparable minimum/maximum envelope, as shown in Fig. 4.

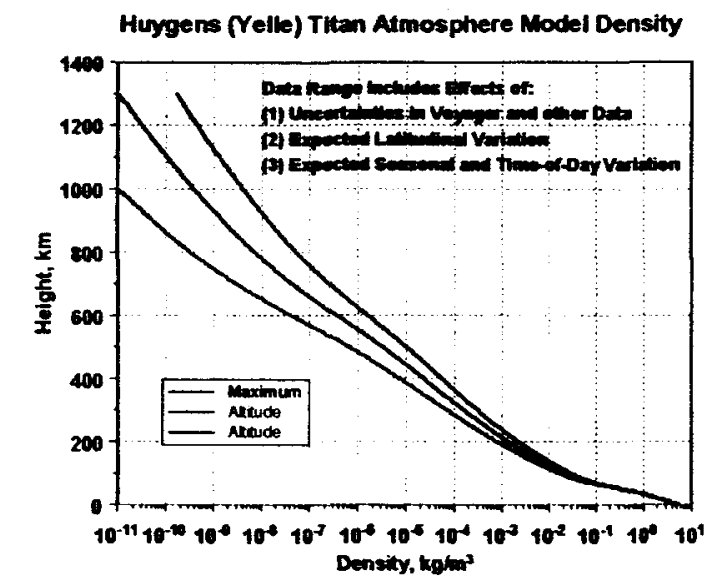

Figure 3: Minimum, average, and maximum density profiles for Titan'

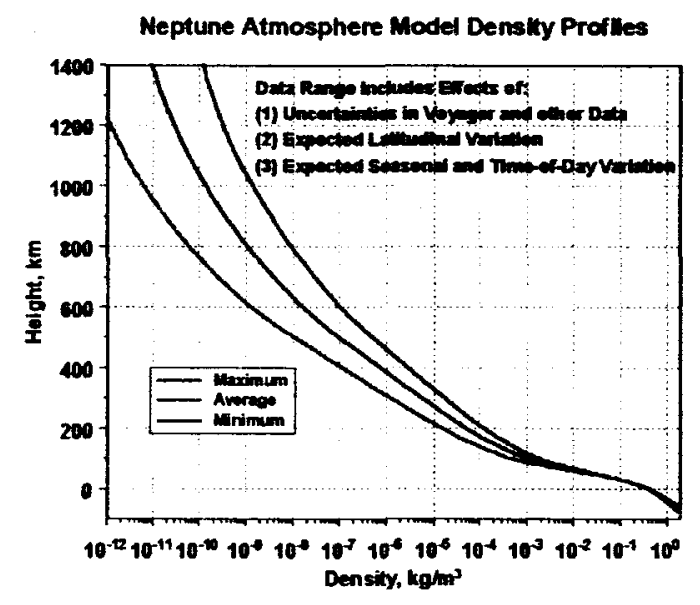

Figure 4: Minimum, average, and maximum density profiles for Neptune from data in Ref. 10.

A single model input parameter (Fminmax) allows the user of Titan-GRAM or Neptune-GRAM to select where within the min-max envelope a particular simulation will fall. Fminmax $=-1,0$, or 1 selects minimum, average, or maximum conditions, respectively, with intermediate values determined by interpolation (i.e. Fminmax between 0 and 1 produces values between average and maximum). Effects such as variation with latitude along a given trajectory path can be computed by user-selected representations of variation of Fminmax with latitude.

Since drag is proportional to density, this is the most important atmospheric parameter for aerocapture. Next most important is height variation of density (as characterized by density scale height). Density scale height is important in determining aerocapture corridor width (entry angle range allowable while still achieving a capture orbit, without "skipping out" or "burning in"). As discussed above, small density scale height means rapid change of density with altitude, which results in low corridor width. Large density scale height implies slow density change with altitude and large corridor width.

Figure 5 compares height profiles of density scale height among the planets and Titan. Aerocapture altitude (c.f. discussion of Fig 2) is indicated by letter $A$ in Fig. 5. This figure shows low density scale height ( 4 to $8 \mathrm{~km}$ ) at aerocapture altitude for the terrestrial planets. Larger scale heights $(\approx 30$ to $50 \mathrm{~km})$ occur at aerocapture altitudes on Neptune and Titan.

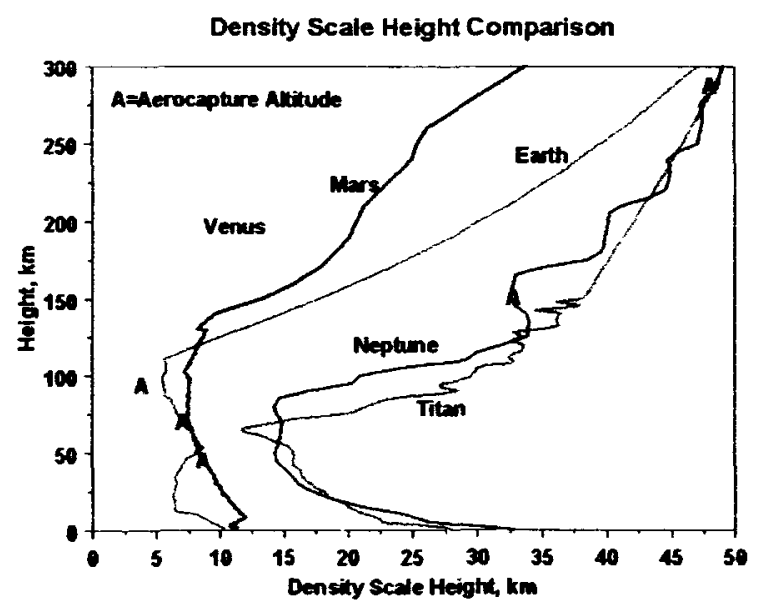

Figure 5: Comparison of atmospheric density scale height among the planets and Titan 


\section{Titan-GRAM GCM Option}

An option has recently been added for using Titan General Circulation Model (GCM) data as input for TitanGRAM. The Titan GCM data used are from graphs in Ref 12. Upper altitudes for the Titan GCM option are computed using a parameterized fit to Titan exospheric temperatures, taken from graphs in Ref 13. Figure 6 gives a height-latitude cross section of density (expressed as percent deviation from the mean) for Voyager encounter date (November 12, 1980, planeto-centric longitude of Sun, $L s=8.8^{\circ}$ ), 00:00 GMT, longitude zero, local solar time 0.7 Titan hours. Figure 7 compares vertical density profiles at latitude zero, local solar time $=1$ hour and 13 hours on Voyager encounter date with Yelle ${ }^{9}$ minimum/maximum density envelope, from Fig. 3. This figure shows that the Titan GCM results correspond fairly closely with Yelle maximum conditions up to about $300 \mathrm{~km}$ altitude, but agree closely with Yelle average conditions (vertical line at 0 in Fig. 7) above about $500 \mathrm{~km}$.

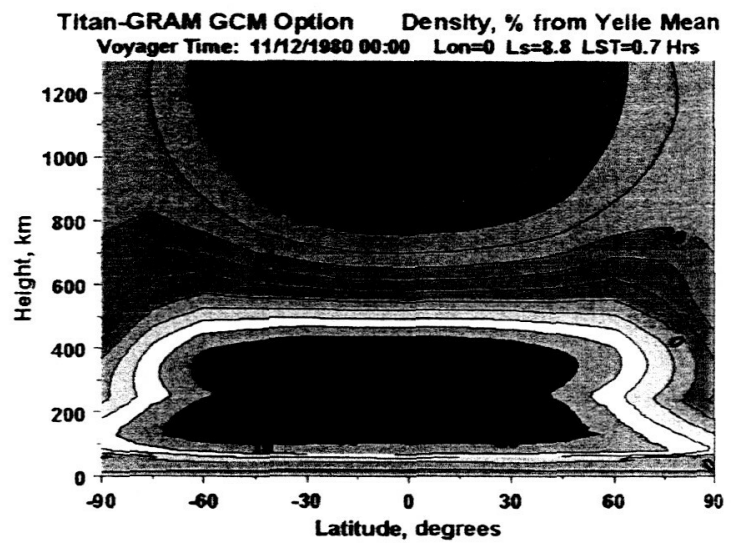

Figure 6: Density (percent deviation from mean) versus height and latitude, using Titan-GRAM GCM option.

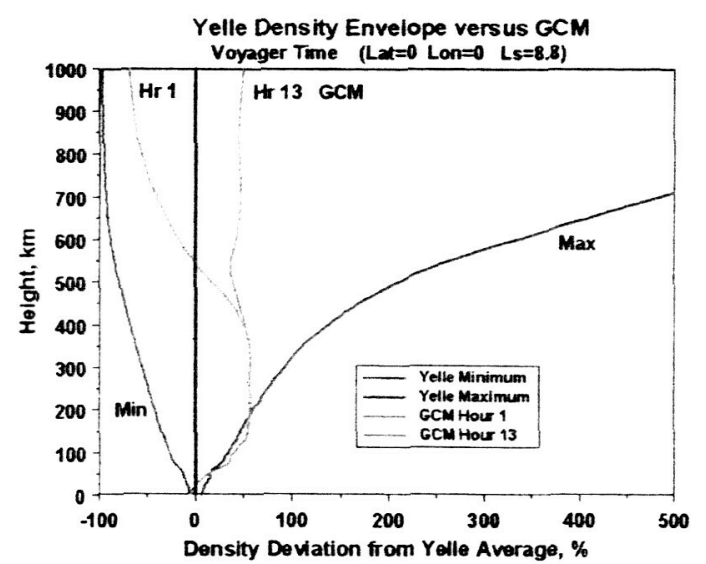

Figure 7: Comparison of two selected TitanGRAM density profiles using GCM option with minimum/maximum envelope from Huygens (Yelle) model'.

\section{Venus-GRAM Development}

Based on the Venus International Reference Atmosphere (VIRA) $)^{11}$, Venus-GRAM is being developed and applied for ongoing Venus aerocapture performance analyses. Figure 8 gives a plot of density (percent deviation from the mean) versus height and latitude from Venus-GRAM. Conditions in Fig. 8 are for $L s=90^{\circ}$ and local solar time 12 Venus hours.

Below about $100 \mathrm{~km}$ altitude on Venus, temperature, density, and density scale height conditions are very uniform with both latitude and time of day. VIRA data below $100 \mathrm{~km}$ altitude vary only slightly with latitude and have no dependence on local solar time. Between 100 and $150 \mathrm{~km}$, VIRA data depend on local solar time (but not latitude). From $150 \mathrm{~km}$ to its top at $250 \mathrm{~km}$, VIRA depends on solar zenith angle, which is affected by both latitude and local solar time.

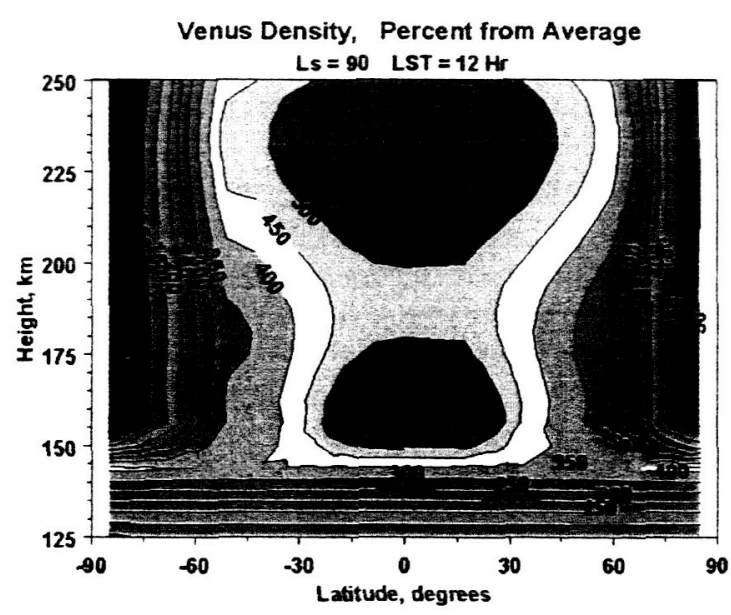

Figure 8: Example height-latitude density cross section from Venus-GRAM.

\section{Perturbation Models}

An important feature of all these engineering-level atmospheric models is their ability to simulate "high frequency" perturbations in density (and winds) due to such phenomena as turbulence and various kinds of 
atmospheric waves. As illustrated in Fig. 9, altitude, latitude, and monthly variations of perturbation standard deviations in Earth-GRAM are based on a large climatology of observations. For Titan and Neptune, perturbation standard deviations are computed from an analytical expression for gravity wave saturation conditions, explained more fully in Ref. 7. As shown in Fig. 9, the resulting vertical profile of standard deviations for Titan and Neptune are not very dissimilar from Earth observations, when expressed as percent of mean density. For Mars, a similar gravity wave saturation relation is used to estimate density perturbation standard deviations, except that effects of significant topographic variation on Mars are also taken into account. Up to about $75 \mathrm{~km}$ altitude, the Mars model density standard deviations are also fairly consistent with Earth observations. By about 100 to $130 \mathrm{~km}$ altitude, Mars model density standard deviations increase to about 20 to $35 \%$ of mean value, consistent with observed orbitto-orbit density variations observed by Mars Global Surveyor and Mars Odyssey.

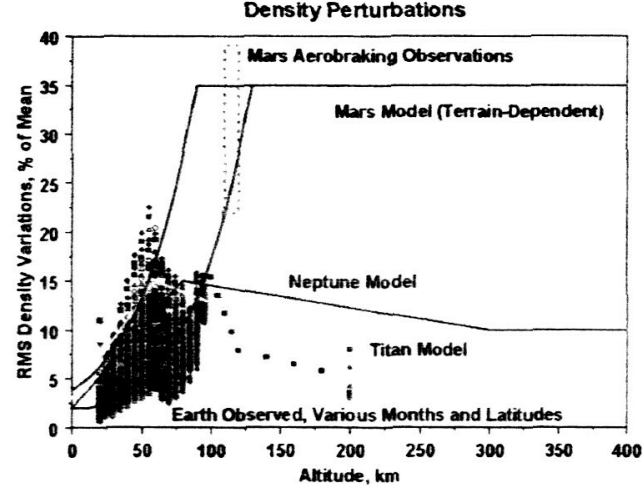

Figure 9: Height variation of density perturbation model standard deviations for Earth, Mars, Titan, and Neptune.

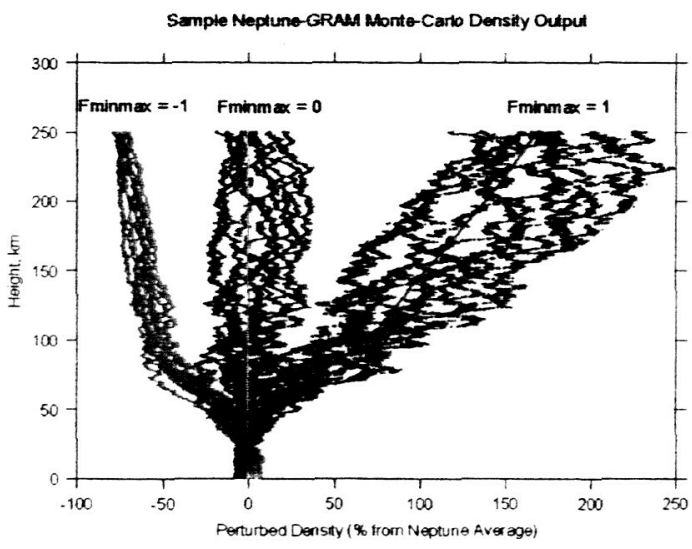

Figure 10: Sample Monte-Carlo density perturbations from Neptune-GRAM, expressed as Dercent deviation from Nebtune mean value.

The design reference mission for which aerocapture was analyzed at Neptune involved capture into a highly eccentric orbit, to allow the simulated orbiter to periodically visit Triton for scientific observations. Ability to successfully aerocapture into such an eccentric orbit depends very significantly on details of Monte-Carlo simulations, such as illustrated in Fig 10. For such an eccentric orbit, there is relatively little margin for error between a captured orbit and one which exceeds escape velocity upon atmospheric exit, a result which could lead to mission failure.

\section{Conclusions}

Engineering-level atmospheric models presented here are suitable for a wide range of mission design, systems analysis, and operations tasks. For orbiter missions, applications include analysis for aerocapture or aerobraking operations, analysis of station-keeping issues for science orbits, analysis of orbital lifetimes for end-of-mission planetary protection orbits, and atmospheric entry issues for accidental break-up and burn-up scenarios. For lander missions to Venus, Mars and Titan, and for Earth-return, applications for these atmospheric models include analysis for entry, descent and landing (EDL), and guidance, navigation and control analysis for precision landing, and hazard avoidance. Perturbation simulation capabilities in these models make them especially useful in doing MonteCarlo analyses for design and testing of guidance, navigation, and control algorithms and for heat loads analysis of thermal protection systems.

\section{Acknowledgments}

The authors gratefully acknowledge support from the NASA/Marshall Space Flight Center In-Space Propulsion Program. Particular thanks go to Bonnie James (MSFC), Manager of the Aerocapture Technology Development Project, to Michelle M. Munk (LaRC/MSFC), Lead Systems Engineer for Aerocapture, and to Melody Herrmann (MSFC), team lead and Mary Kae Lockwood (LaRC), technical lead for the Titan/Neptune Systems Analysis study. Model user feedback and suggestions from the following are also greatly appreciated: Dick Powell, Brett Starr, and David Way (NASA LaRC), and Claude Graves, Jim Masciarelli, Lee Bryant, Tim Crull, and Tom Smith (NASA JSC). External review comments from Prof. Darrell Strobel (Johns Hopkins University) were especially helpful. 


\section{References}

'Justus, C. G., and Johnson, D. L., "The NASAMSFC Global Reference Atmospheric Model - 1999 Version (GRAM-99)", NASATTM-1999-209630, 1999.

${ }^{2}$ Justus, C.G., Duvall, A. L., and Johnson, D. L., "Earth Global Reference Atmospheric Model and Trace Constituents", 34th COSPAR Scientific Assembly, Houston, Texas, Invited Paper C4.1-0005-02, October, 2002.

${ }^{3}$ Justus, C. G., and Johnson, D. L., “Mars Global Reference Atmospheric Model 2001 Version (Mars-GRAM 2001) Users Guide”, NASATM-2001-210961, April, 2001.

"Justus, C. G., Duvall, A. L., and Johnson, D. L., "Mars-GRAM Validation with Mars Global Surveyor Data", 34th COSPAR Scientific Assembly, Houston, Texas, Paper C3.3-0029-02, October, 2002.

5Justus, C. G., Duvall, A. L., and Johnson, D. L., “Global MGS TES Data and Mars-GRAM Validation”, 34th COSPAR Scientific Assembly, Houston, Texas, Paper C4.2-0005-02, October, 2002.

6Justus, C. G., Duvall, A. L and Johnson, D. L. "Mars Global Reference Atmospheric Model (Mars-GRAM) and Database for Mission Design", International Workshop on Mars Atmosphere Modeling and Observations, Granada, Spain, January, 2003.

'Justus, C.G., Duvall, A. L, and Johnson, D. L., "Engineering-level model atmospheres for Titan and Neptune", 39th AIAAASME/SAE/ASEE Joint Propulsion Conference, Huntsville, Alabama, Paper AIAA-2003-4803, July, 2003.

${ }^{8}$ Justus, C.G., Duvall, A. L, and Keller, V. W., "Engineering-level model atmospheres for Titan and Mars", International Workshop on Planetary Probe Atmospheric Entry and Descent Trajectory Analysis and Science, Lisbon, Portugal. October, 2003.

9Yelle, R.V. , Strobell, D. F., Lellouch, E, and Gautier, D., “Engineering Models for Titan's Atmosphere”, in Huygens Science, Payload and Mission, ESA SP-1177, August, 1997.

${ }^{10}$ Cruikshank, D.P. (ed), Neptune and Triton, University of Arizona Press, Tucson, 1995.

"Kliore, A. J., Moroz, V. L., and Keating, G. M. (eds.), "The Venus International Reference Atmosphere", Advances in Space Research, vol. 5, no. 11, 1985, Pergamon Press, Oxford, 1986, pp. 1-304.

${ }^{12}$ Hourdin, F., Talagrand, O., Sadourny, R., Courtin, R., Gautier, D., and McKay, C.P., "Numerical simulation of the general circulation of the atmosphere of Titan", Icarus, vol. 117, no. 2, Oct. 1995, pp. 358-74.

${ }^{13}$ Mueller-Wodarg, I. C. F., "The Application of General Circulation Models to the Atmospheres of Terrestrial-Type Moons of the Giant Planets", in Comparative Amospheres in the Solar System, American Geophysical Union, 2002. 